\title{
Atomic force microscopy of the nucleolus of Ginkgo biloba
}

María Lourdes Segura-Valdez ${ }^{1}$, Ana Paulina Mendoza von der Borch ${ }^{1}$, Sarai de Jesús Cruz-Gómez ${ }^{1}$ and Luis F. Jiménez-García ${ }^{2}$

${ }^{1}$ UNAM, Coyoacán, Distrito Federal, Mexico, ${ }^{2}$ UNAM, Coyoacán, Mexico

In eukaryotes, the nucleolus is the major site for the cytoplasmic ribosome biogenesis. AT the ultrastructural level, the nucleolus is composed of three main elements named fibrillar centers, dense fibrillar component and granular component. Many nucleoli of many species have been analyzed with the transmission electron microscope and all of them display this type of structure. Here we have used atomic force microscopy to study the fine structure of the nucleolus of the tree Ginkgo biloba and contrast the results to those obtained by electron microscopy. Small fragments of buds from G. biloba were processed as for standard transmission electron microscopy. Briefly, samples were fixed in glutaraldehyde, postfixed in osmium tetroxide, dehydrated in a series of graded ethano1, and propylene oxide and embedded in epoxy resin polymerized at 60 degrees. Semithin sections of about $250 \mathrm{~nm}$ width mounted on glass slides were observed with an atomic force microscope operating in contact mode. Other sections were stained with toluidine blue. Thin sections of some $50 \mathrm{~nm}$ width were contrasted with uranyl acetate and lead citrate and then were observed with a transmission electron microscope working at $80 \mathrm{kV}$. Results show that nucleoli are present as large rounded figures within the cell nuclei as observed by bright field light microscopy. By transmission electron microscopy, nuclei of G. biloba display nucleoli where fibrillar centers, dense fibrillar component and granular components are showed, Nucleolar granules about $25 \mathrm{~nm}$ in diameter are distinguished from other fibrillar elements. Using the atomic force microscopy working in contact mode, nucleoli are observed where high profiles we recognized as fibrillar component. Granular component displays different high profiles. However, we have been unable to observe fibrillar centers, that are elusive. We conclude that internal nucleolar ultrastructure can be analyzed with atomic force microscopy working in contact mode, by using unstained semithin sections mounted on glass slides. 


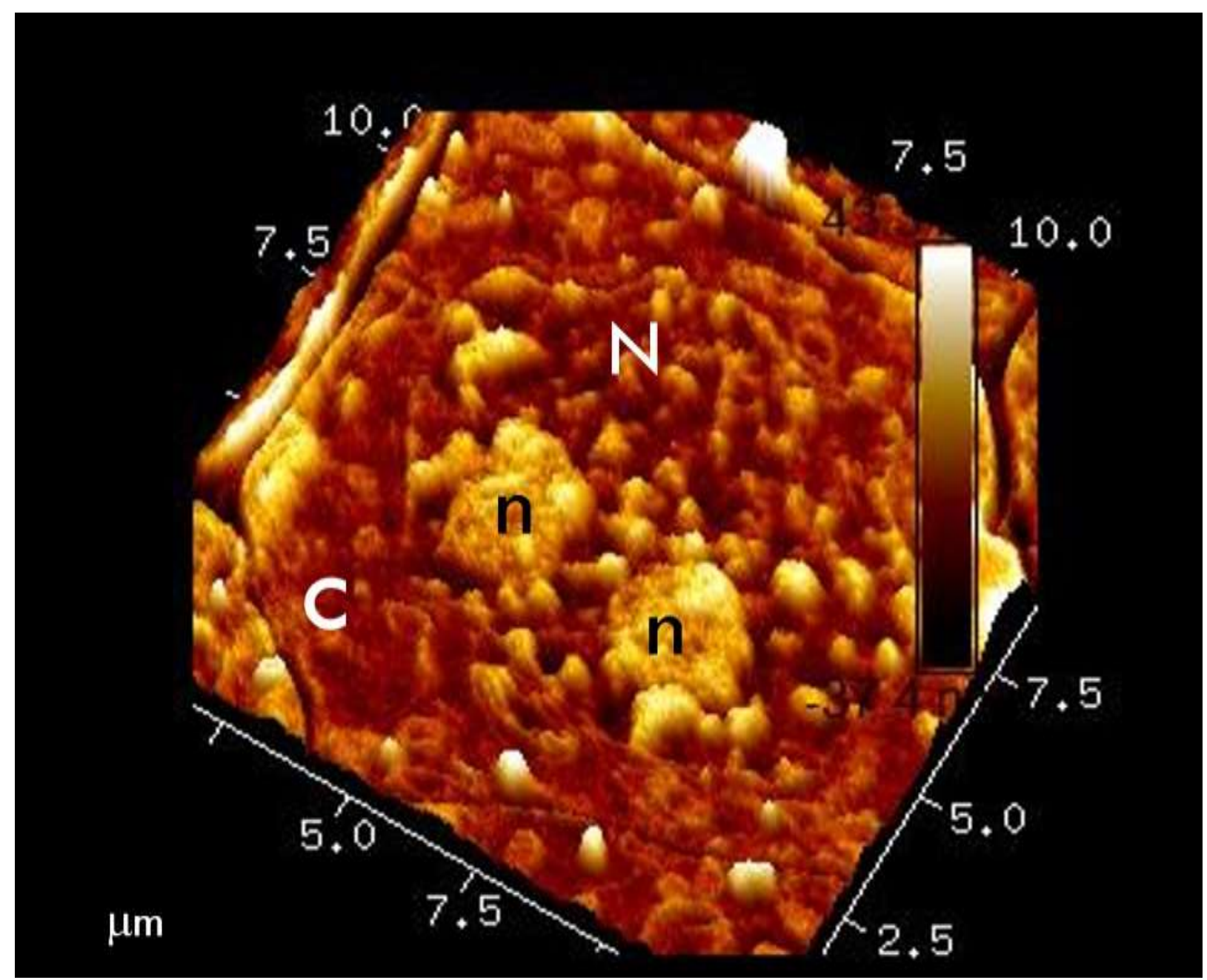

Figure 1. Atomic force micrograph of nucleoli of Ginkgo biloba. C, cytoplasm; N, nucleus; n, nucleoli

\section{References}

Jiménez-Ramírez J. et al. (2002).Biol Cell 94: 511-518 